\title{
Onset of thermal convection of a fluid-saturated porous medium in a vertical cylinder with mixed heating on lateral wall
}

\author{
I-Chung Liu, Yen-Tsung Chang, Jawali C. Umavathi
}

\begin{abstract}
In this paper we examine the thermal-convective stability of a fluid-saturated porous medium filled within a vertical cylindrical container heated from below. The upper and lower surfaces of the cylinder are impermeable and are kept at constant but different temperatures. The lateral sidewall is impermeable and subject to mixed heating which is characterized by the Biot number. The threshold of Rayleigh number, above which the small disturbance becomes unstable, is sought from the characteristic equation. Our results show that the preferred mode at onset is always axisymmetric for all aspect ratios when Biot number approaches infinitely, and it becomes non-monotonic variation with respect to aspect ratio when Biot number is finite.
\end{abstract}

Keywords - thermal convection, porous medium, Newtonian heating, cylindrical container, Biot number.

\section{Introduction}

The study of natural convection in a fluid-saturated porous medium serves as an important field for the flow in thermal reservoir, thermal insulation design, geothermal energy restoration, and many other heat transfer processes of industrial applications. Horton and Rogers [1] and Lapwood [2] initiated the classical Rayleigh-Benard convection in an infinite horizontal layer of a fluid-saturated porous medium heated from below and they obtained a threshold value of Rayleigh number $4 \pi^{2}$, above which the small disturbance may cause cellular motion. This thermal motion can not occur for infinitesimal disturbances when the Rayleigh number is below $4 \pi^{2}$. The mode of convection just above the critical state is of no preferred direction in the case of infinite porous layer. However the critical Rayleigh number $\left(R a_{c}\right)$ and the preferred mode behave quite different in a finite domain from those in infinite porous layer. Beck [3] investigated the convection in a box of porous material saturated with viscous fluid and concluded that the roll (a cell with only two non-zero velocity components) is not the only cellular mode and the roll axis direction is such that there is the greatest degree of "squareness" in the cross section of each roll. He gave a picture showing the complex variation of critical Rayleigh number and preferred convection mode with respect to the two lateral dimensions.

I-Chung Liu

National Chi Nan University

Taiwan, R.O.C.

Yen-Tsung Chang

National Chi Nan University

Taiwan, R.O.C
The critical Rayleigh number is always greater than $4 \pi^{2}$, the value corresponding to unbounded fluid-saturated porous medium. When the lateral dimension becomes large, the critical Rayleigh number quickly approaches to $4 \pi^{2}$. Beck [3] also proved that the critical Rayleigh number from linear analysis meets that from nonlinear analysis, thus no subcritical instability is possible.

Zebib [4] applied a linear stability analysis to the natural convection in a cylinder of water saturated porous medium with impermeable and isothermal conditions at both lower and upper surface, and impermeable and insulated lateral wall. His results illuminated that $\mathrm{Ra}_{\mathrm{c}}$ was a combination of "cusps" with respect to aspect ratio defined as the ratio of radius to height of the cylindrical container. The cusp is characterized by the mode $(m, n)$ where $\mathrm{m}$ and $\mathrm{n}$ represent the azimuthal and radial modes, respectively. The preferred mode with respect to aspect ratio in most cases shows nonaxisymmetric type $(m \neq 0)$ rather than symmetric $(m=0)$ even the container is symmetric. In addition the convection motion is truly three dimensional. Later on Wang [5] considered Zebib's case [4] except for constant heat flux condition at lower surface, simulating the bottom heated by an electric heating device. An exactly the same mode change sequence was found, as that in Zebib [4], though the deviation in values of critical Rayleigh number prevailed. The minimum of Rac is 27.0976 occurring at several values of aspect ratio. This minimum is also prevalent when aspect ratio becomes very large, which is also the stability criterion for an infinite layer with constant heat flux from below. Bau and Torrance [6] also extend Zebib's situation [4] to a permeable top surface, which reflects to the case of fluid overlaying the porous medium; i.e. the constant pressure case. Wang [7] re-examined his study [5] by using permeable top condition like Bau and Torrance [6], and he found that Rac approaches to 17.6537 when aspect ratio becomes unbounded. Kubitschek and Weidman [8] analyzed thermal convection of fluid-saturated porous medium in a vertical cylinder with a more general thermal heating condition; that is, the mixed heating or so-called Newtonian heating. When the Biot number becomes negligible, [8] reduces to Zebib case [4], and if Biot number increases without bound, [8] degenerates to Wang's case [5]. The mode change sequences in [4-8] appear in the same manner because the azimuthal eigen-mode is completely determined by the lateral boundary condition, or the impermeable and insulating sidewall. The assumption for insulating sidewall exactly leads to the same mathematical condition as the condition for impermeable sidewall. Consequently, the original fourth-order ordinary differential equation degenerates to a second-order one.

In practical situation, not all lateral conditions of above mentioned problem happen to be impermeable and insulated. Haugen and Tyvand [9] explored the thermal convection of fluid-saturated porous medium in a vertical 
cylinder heated from below, specifying constant temperature at lateral surface rather than the insulating case studied before [3-8]. The constant temperature sidewall means that the lateral surface is perfectly conducting and the heat in the cylinder is permitted to leave from the lateral boundary. This thermal condition generally causes the onset of thermal convection to be delayed together with a higher critical Rayleigh number, and the prevailed mode changes present in a different manner as those in insulating case. The convection starts in the form of axisymmetric mode with all aspect ratios rather than the "cusp" variation for insulating lateral wall case [3-8].

Motivated by previous literature we study the thermal convection of a homogeneous and isotropic porous medium saturated with a viscous fluid in a vertical cylinder, taking into account the later wall being impermeable and perfectly conducting. The variation of critical Rayleigh number and preferred mode change sequence are explored with respect to aspect ratio and Biot number. The results are presented in form of tables and figures.

\section{Problem Formulation}

We consider a vertical cylindrical container filled with a fluid saturated in a homogeneous and isotropic porous material. The height of the container is $H$ and the radius is $r_{0}$ with the aspect ratio being $a=r_{0} / H$. A cylindrical coordinate $(r, \phi, z)$ is employed with $z$-axis pointed upwards, opposed to the direction of gravity, as shown in Fig. 1. The velocity components $(u, v, w)$ are in the directions of $(r, \phi, z)$, respectively. The sidewall of cylindrical container is impermeable and subject to Newtonian heating with the heat transfer coefficient $h$. The bottom $z=0$ is maintained at constant temperature $T_{0}$ and the top surface $z=H$ is kept at constant temperature $T_{1}$, with $\Delta T=T_{0}-T_{1}$. In a quiescent state, the basic velocity and temperature of take the form

$$
\mathbf{u}_{\mathrm{b}}=(0,0,0), T_{\mathrm{b}}=T_{0}-\Delta T z / H,
$$

where $b$ represents the quantity of the basic state. The basic temperature with a dimensional gradient $-\Delta T / H$ is assumed, which is due to pure conduction. The boundary condition at the side wall should be

$$
r=\mathrm{r}_{0}, k \partial T / \partial r=h\left[T_{\infty}-T\right],
$$

where $T_{\infty}$ is the temperature outside the lateral wall. The condition of (2) states that the external heat flux delivered to the fluid-saturated porous medium at the lateral wall is $q=h\left[T_{\infty}-T\left(r_{0}\right)\right]$.

The dimensionless equations governing the DarcyBoussinesq fluid saturated in a porous medium admit

$$
\begin{gathered}
\nabla \cdot \mathbf{u}=0, \\
\mathbf{u}+\nabla P-R a T \mathbf{k}=0, \\
\partial T / \partial t+\mathbf{u} \cdot \nabla T=\nabla^{2} T,
\end{gathered}
$$

where $\boldsymbol{u}$ is the velocity vector, $T$ is temperature, $P$ is the pressure and $t$ is time. The Rayleigh number is defined as $R a=g \alpha K \Delta T H / v \kappa_{\mathrm{m}}$. Equations (3)-(5) have been made dimensionless using $\kappa_{\mathrm{m}} / H, H,\left(\rho c_{\mathrm{p}}\right)_{\mathrm{m}} H^{2} / \kappa_{\mathrm{m}}, \Delta \mathrm{T}$, and $\rho_{0} v \kappa_{\mathrm{m}} / K$ as the scales for velocity, length, time, temperature, and pressure, respectively. The physical quantities are: $g$ the gravitational acceleration, $\alpha$ the coefficient of thermal expansion, $c_{\mathrm{p}}$ the specific heat at constant pressure, $v$ the kinematic viscosity, $\kappa$ the thermal diffusivity, $K$ the permeability of the mixture, and $\rho_{0}$ the reference temperature at $T_{0}$. The subscript $m$ denotes the properties belonging to the mixture of fluid and porous medium.

We perturb the basic quantities with

$$
\begin{aligned}
& \mathbf{u}=\mathbf{u}_{b}(z)+\mathbf{u}^{\prime}(r, \phi, z, t), T=T_{b}(z)+\theta^{\prime}(r, \phi, z, t), \\
& P=P_{b}(z)+p(r, \phi, z, t) .
\end{aligned}
$$

Substituting (6) in (3)-(5), eliminating the pressure, and neglecting higher-order terms from the resultant equations, we obtain the linearized version with dropping the primes:

$$
\begin{aligned}
& \nabla^{2} w-R a \nabla_{1}^{2} \theta=0, \\
& w+\nabla^{2} \theta=0,
\end{aligned}
$$

The corresponding boundary conditions take the form

$$
\begin{aligned}
& w=0, \theta=0 \quad \text { at } z=0,1, \\
& u=0, \partial \theta / \partial r+B i \theta=0 \text { at } r=a,
\end{aligned}
$$

where $a=r_{0} / H$ is the aspect ratio, and $B i=h H / k$ is the Biot number. Equation (9) results from the impermeable and perfectly conducting conditions at both upper and lower surfaces, whereas (10) reflects to the consequence of impermeable and mixed heating at lateral wall. In the limiting case of large Biot number, we recover the case of Haugen and Tyvand [9] for isothermal cylinder wall, whereas the situation reduces to Zebib [4] for constant heat flux lateral wall when Biot number becomes negligibly small. We note that the onset of convection can be proved as stationary type and thus the time-derivative term is absent. The stability problem (7)-(10) are coupled in velocity and temperature. We may decouple them to the form

$$
\begin{gathered}
\nabla^{4} w+R a \nabla_{1}^{2} w=0 \\
\nabla^{4} \theta+\operatorname{Ra} \nabla_{1}^{2} \theta=0
\end{gathered},
$$

We employ a sinusoidal function $\sin (n \pi z)$ to satisfy the boundary conditions (9). Thus the horizontal Lapalcian operator $\nabla_{1}^{2}$ may be replaced by $\nabla^{2}+n^{2} \pi^{2}$, where $n$ is a positive integer. Then (11) and (12) become

$$
\begin{aligned}
& \nabla^{4} w+\operatorname{Ra} \nabla^{2} w+\operatorname{Ra} n^{2} \pi^{2} w=0 \\
& \nabla^{4} \theta+\operatorname{Ra} \nabla^{2} \theta+\operatorname{Ra} n^{2} \pi^{2} \theta=0 .
\end{aligned}
$$

The most unstable mode prevails for the lowest mode in the vertical direction, we set $n=1$. Factorizing above two equations leads to

$$
\begin{aligned}
& \left(\nabla^{2}+b\right)\left(\nabla^{2}+c\right) w=0, \\
& \left(\nabla^{2}+b\right)\left(\nabla^{2}+c\right) \theta=0,
\end{aligned}
$$

where the parameters satisfy the relation:

$$
b+c=R a, b c=\pi^{2} R a .
$$

The constants $b$ and $c$ can be explicitly solved and they are both positive if $R \mathrm{a} \geq 4 \pi^{2}$. We now deal with the temperature equation only. The eigenfunctions of (16) must satisfy the two Helmholtz equations

$$
\begin{aligned}
& \left(\nabla^{2}+b\right) \theta=0, \\
& \left(\nabla^{2}+c\right) \theta=0 .
\end{aligned}
$$

The total solution of the present convection problem should be a linear combination of solution for each Helmholtz equation, and it will be the complete solution if $b$ and $c$ are different. If $b=c$, we have $R a=4 \pi^{2}$ which is exactly the $R a_{\mathrm{c}}$ for thermal convection of an infinite fluid layer saturated in porous medium (Horton and Rogers [1], Lapwood [2]) or the $R a_{c}$ in the limiting case of large aspect ratio with 
insulating walls (Beck[3], Zebib[4]). We note that the $R a_{c}$ is the smallest one with respect to all possible wavenumbers for an infinite fluid layer without lateral boundaries. However, it will be the smallest one for discrete wavenumber depending upon the geometry of the container when lateral boundary is concerned. Heat conduction at the lateral walls usually takes away the heat and thus delays the onset of convection, leading to critical Rayleigh number $R a=4 \pi^{2}$. The analytical approaches con-firmed this argument, the readers are referred to Zebib [4], Kubitschek and Weidman [8], Haugen and Tyvand [9], Nilsen and Storesletten [10]. Incorporated with the curl of Darcy's law, the first boundary condition in (10) becomes

$$
\frac{\partial w}{\partial r}-R a \frac{\partial \theta}{\partial r}=0 \text { at } r=a
$$

Taking differentiation of heat equation with respect to $r$ yields

$$
\frac{\partial w}{\partial r}+\frac{\partial}{\partial r} \nabla^{2} \theta=0
$$

We arrive at a single expression for velocity and temperature condition from (20) and (21)

$$
\frac{\partial}{\partial r}\left[\nabla^{2} \theta+R a \theta\right]=0 \text { at } r=a \text {. }
$$

\section{Method of Solution}

The eigenvalue problem for temperature perturbation composed of (18), (19), (10) and (22) is solved by using separation of variables. We assume two eigen-functions corresponding to the two Helmholtz equations in the form

$$
\begin{aligned}
& \theta_{1}=J_{m}(p r) \cos m \phi \sin \pi z, \\
& \theta_{2}=J_{m}(q r) \cos m \phi \sin \pi z,
\end{aligned}
$$

where $J_{m}$ is the Bessel function of order $m$ of the first kind, and $m$ is a non-negative integer. The two wavenumbers $p>0$ and $q>0$ are related to $b$ and $c$ through the two Helmholtz equations by:

$$
b=p^{2}+\pi^{2} \text { and } c=q^{2}+\pi^{2} .
$$

They can be recast in terms of $\mathrm{Ra}$ as

$$
p^{2}+q^{2}=R a-2 \pi^{2} \text { and } p q=\pi^{2} .
$$

The total solution will now be a linear combination of the two eigen-functions $\theta_{1}$ and $\theta_{2}$ as

$$
\theta=A_{1} \theta_{1}+A_{2} \theta_{2}
$$

where $A_{1}$ and $A_{2}$ are constants.

Substituting (27) into (10) and (22) and utilizing (26), we get

$$
\left[\begin{array}{cc}
p J_{m}^{\prime}(p a)+B i J_{m}(p a) & q J_{m}^{\prime}(q a)+B i J_{m}(q a) \\
J_{m}^{\prime}(p a) & J_{m}^{\prime}(q a)
\end{array}\right]\left[\begin{array}{l}
A_{1} \\
A_{2}
\end{array}\right]=0
$$

where the primes denote differentiation with respect to their respective argument. To have non-trivial solution for $A_{1}$ and $A_{2}$, we can obtain the characteristic equation by vanishing the determinant of (28)

$$
\begin{aligned}
& (p-q) J_{m}^{\prime}(p a) J_{m}^{\prime}(q a)+ \\
& B i\left[J_{m}(p a) J_{m}^{\prime}(q a)-J_{m}(q a) J_{m}^{\prime}(p a)\right]=0
\end{aligned}
$$

The corresponding temperature can now be expressed as

$$
\begin{aligned}
\theta= & \frac{A_{1}}{J_{m}^{\prime}(q a)}\left[J_{m}^{\prime}(q a) J_{m}(p r)\right. \\
& \left.\quad-J_{m}^{\prime}(p a) J_{m}(q r)\right] \cos m \phi \sin \pi z
\end{aligned}
$$

Two limiting cases for above equation can be deduced. By letting $B i \rightarrow \infty$ or the perfectly heat-conducting wall, the condition (29) becomes (Haugen and Tyvand [9])

$$
J_{m}(q a) J_{m}^{\prime}(p a)-J_{m}(p a) J_{m}^{\prime}(q a)=0
$$

For negligible Biot number $(B i \rightarrow 0)$, i.e. the case of insulating wall, we have

$$
(p-q) J_{m}^{\prime}(p a) J_{m}^{\prime}(q a)=0,
$$

which can be reduced to Zebib [4].

We may replace $q$ by $\pi^{2} / p$ from (26). Once the solution of (29) is searched numerically, the corresponding Rayleigh number is given by

$$
R a=p^{2}+\pi^{4} / p^{2}+2 \pi^{2}=\left(p+\pi^{2} / p\right)^{2} .
$$

Regarding (33), the smallest allowable Rayleigh number is the critical Rayleigh number and it takes the value of $4 \pi^{2}$, which is exactly the critical Rayleigh number for infinite porous layer. This condition occurs only when $p=q=\pi$. However, this is not the case if $a$ is finite. Since the Rayleigh number is an increasing function of $p$ when $q$ is greater than $\pi$, in view of (33), the search for critical Rayleigh number is equivalent to finding the smallest possible value of $p$ requiring the zero determinant. To verify our numerical result, some values of $p$ for several azimuthal modes $m$, aspect ratio $a$ and infinite Biot number $B i \rightarrow \infty$ are compared with those of Haugen and Tyvand [9] (not shown). The agreement is excellent. Table 1 gives the results of $R a_{\mathrm{c}}$ and $(m, n)$ for a specific $a$ at $B i=0$. These tables show an excellent agreement compared with existing data. We note that when $B i \rightarrow 0$, i.e. the insulating wall, the critical Rayleigh number shows a non-monotonic variation of aspect ratio $a$ (Zebib [4]), but it shows a smooth relation with $a$ when $B i \rightarrow \infty$, i.e. the perfectly heat-conducting wall (Haugen and Tyvand [9]). In addition, the Rayleigh number for insulating case is always smaller than that for perfectly heat-conducting case, implying that heat may lose through the perfectly heat-conducting boundary and result in a higher Rayleigh number.

Using the relation of $J_{m}^{\prime}(x)=\left[J_{m-1}(x)-J_{m+1}(x)\right] / 2$, the characteristic equation (29) becomes

$$
\begin{gathered}
(p-q)\left[J_{m-1}(p a)-J_{m+1}(p a)\right]\left[J_{m-1}(q a)-J_{m+1}(q a)\right] / 2 \\
+B i\left\{J_{m}(p a)\left[J_{m-1}(q a)-J_{m+1}(q a)\right]\right. \\
\left.-J_{m}(q a)\left[J_{m-1}(p a)-J_{m+1}(p a)\right]\right\}=0
\end{gathered}
$$

For axisymmetric mode $m=0$, the characteristic equation reduces to 
Proc. of The Fifth Intl. Conf. On Advances in Applied Science and Environmental Technology - ASET 2016 Copyright ( I Institute of Research Engineers and Doctors, USA .All rights reserved.

ISBN: 978-1-63248-106-1 doi: 10.15224/ 978-1-63248-106-1-33

$$
\begin{aligned}
& (p-q) J_{1}(p a) J_{1}(q a)+ \\
& B i\left[J_{0}(q a) J_{1}(p a)-J_{0}(p a) J_{1}(q a)\right]=0
\end{aligned}
$$

The velocity component $w$ can be obtained from (8) and (27) as

$$
\begin{aligned}
& w=\frac{A_{1}}{J_{m}^{\prime}(q a)}\left[\left(\pi^{2}+p^{2}\right) J_{m}^{\prime}(q a) J_{m}(p r)\right. \\
& \left.-\left(\pi^{2}+q^{2}\right) J_{m}^{\prime}(p a) J_{m}(q r)\right] \cos m \phi \sin \pi z
\end{aligned}
$$

The stream function can be calculated through the relation

$$
\partial \psi / \partial r=r w
$$

For axisymmetric mode $m=0$, the stream function becomes

$$
\psi=\frac{A_{1}(p+q) r}{J_{1}(q a)}\left[J_{1}(q a) J_{1}(p r)-J_{1}(p a) J_{1}(q r)\right] \sin \pi z
$$

where the integral $\int x J_{0}(x) d x=x J_{1}(x)$ and the identity $J_{0}(x)=-J_{1}(x)$ have been employed. The corresponding radial velocity component in the case of axial-symmetry is given by

$$
\begin{aligned}
u= & -\frac{1}{r} \frac{\partial \psi}{\partial z}=-\frac{A_{1} \pi(p+q)}{J_{1}(q a)} \\
& {\left[J_{1}(q a) J_{1}(p r)-J_{1}(p a) J_{1}(q r)\right] \cos \pi z }
\end{aligned}
$$

\section{Results and Discussions}

We consider the onset of thermal convection of a fluidsaturated porous medium in a vertical cylinder subject to impermeable and constant temperature at both lower and upper surfaces. The balance between heat flux $-k \partial T / \partial r$ and external heat rate delivered to the porous matrix $h\left(T_{\infty}-T\right)$ at side wall is assumed. The characteristic equation (29) for the present thermal convection problem possesses the functional form

$$
F(R a, B i, a, m, n)=0
$$

that can be evaluate numerically over all modes $(m, n)$ to determine the critical Rayleigh number $R a_{\mathrm{c}}$ for fixed $a$ and Bi. Here mode $(m, n)$ is defined as a combination of azimuthal mode and radial mode, respectively. Suggested by Kubitschek and Weidman [8], we examine the thermal stability characteristics for selected $B i$ in the range $10^{-4} \leqslant B i$ $\leqslant 10^{4}$ covering the aspect ratio $0<a \leqslant 3$ with a fine grid spacing 0.001 . We plot the variation of $R a_{\mathrm{c}}$ as a function of $a$ and $B i=10^{-4}, 0.1,1,10$, and $10^{4}$ in the stability curves shown Fig. 2.

The lowest curve at $B i=10^{-4}$ behaves in a close agreement to the result given in Fig. 1 of Zebib [4] which is subject to impermeable and insulating conditions at the lateral wall. The $R a_{\text {c }}$ presents a cusp-like variation with respect to $a$ and the smallest $R a_{\mathrm{c}}$ approaches $4 \pi^{2}$ as $a \rightarrow \infty$. The cusp has discontinuities in derivative of curve in $R a_{\mathrm{c}} \sim a$ diagram, separated by two consecutive modes $(m, n)$ and $\left(m^{*}, n^{*}\right)$, showing the non-monotonic relation between $R a_{\mathrm{c}}$ and $a$. The mode change $(m, n)$ from slender cylinder (small $a$ ) to wide one $(a \approx 3)$ takes on the sequence: $(1,1),(2,1),(0,1),(3,1)$, $(4,1),(1,2),(5,1),(2,2),(0,2),(6,1),(3,2),(1,3),(7,1)$ and $(4,2)$ with the corresponding local maximum (possibly) and aspect ratio between modes change $\left(R a_{\mathrm{c}}, a\right)$ being at $(0.751$, 41.9303), (1.089, 39.9798), (1.278, 39.5603), (1.505, 40.0265), (1.693, 39.4789), (1.862, 39.8166), (2.088, 39.4979), (2.184, 39.4891), (2.310, 39.5222), (2.469, $39.5211),(2.633,39.5177),(2.726,39.4790)$ and $(2.841$, 39.5394).

Other mode change sequences and corresponding $\left(R a_{\mathrm{c}}, a\right)$ for various $B i$ are given in Table 3. We observe that the number of modes appeared decreases in the region $0<a \leqslant 3$. The modes $(6,1)$ and $(7,1)$ are absent for case $B i=0.1$ compared to the case $\mathrm{Bi}=10^{-4}$. Moreover the nonaxisymmetric mode $(4,1)$ shifts from $(1.505,40.0265)$ to $(2.841,39.6907)$ at $B i=10^{-4}$, which reflects to the fact that some specific modes do not really disappear in the stability diagram. They would possibly occur at larger value of $a$ when Biot number increases. The mode change sequence becomes $(1,1)$ (not shown in Fig. 2), $(0,1),(1,2),(0,2),(1,3)$ and $(0,3)$ as $B i$ reaches the value 10 . One may acquire another fact from Fig. 2 that the critical Rayleigh number at large $B i$ is always larger than that at small $B i$ when $a$ keeps fixed. The thermal condition approaches to insulating condition when $B i$ is negligible and to perfectly heatconducting condition when $B i$ is very large. This is because that heat is not permitted to leave from the fluid to the wall for insulating condition $(B i=0)$ and is allowed to transfer across the wall for increasing $B i$. Thus the potential to destroy the thermal equilibrium becomes weaker when the Biot number raises, and consequently results in a higher value of $R a_{\mathrm{c}}$.

The highest curve of $R a_{\mathrm{c}}$ at $\mathrm{Bi}=10^{4}$ depicts a smoothly decreasing function of $a$ rather than the cusp-like variation at $B i=10^{-4}, 0.1,1$ and 10 . We find that the preferred mode of convection is axisymmetric $(m=0)$ for all range of $0<a \leq 3$ except that the non- axisymmetric modes $(m=1)$ are present at very narrow patches of $a \in(1.637,1.663)$ and $(2.673$, 2.703) approximately. Other non-axisymmetric modes do not prevail in our computational range. Haugen and Tyvand [9] gave a mathematical analysis to confirm that only axisymmetric mode of convection is prevalent in slendercylinder limit for perfectly heat-conducting side wall. The smooth relation between $R a_{\mathrm{c}}$ and $a$ was found by Nilsen and Storesletten [10] for thermal convection in a twodimensional box with conducting walls and also verified by Rees and Lage [11] for natural convection in a vertical porous insulation layer. The case at $B i=10^{4}$ also presents a nearly smooth relation between $R a_{\mathrm{c}}$ and $a$ because large $B i$ corresponding to perfectly heat-conducting case. When $B i$ takes the value of infinity, our results agree with Haugen and Tyvand [9].

\section{v. Conclusion}

In this study we have examined the onset of thermal convection of a vertical circular porous cylinder with impermeable and mixed heating at cylindrical wall. Owing to assumption of mixed heating at sidewall, our results reduce to Zebib [4] for insulating case when $B i \rightarrow 0$ and to 
Proc. of The Fifth Intl. Conf. On Advances in Applied Science and Environmental Technology - ASET 2016 Copyright (C) Institute of Research Engineers and Doctors, USA .All rights reserved.

ISBN: 978-1-63248-106-1 doi: 10.15224/ 978-1-63248-106-1-33

Haugen and Tyvand [9] for perfectly conduction case when $B i \rightarrow \infty$. The following are main findings:

(1) The stability curve presents a cusp-like variation with respect to aspect ratio for finite Biot number but it becomes a smooth variation for very large Biot number; that is, nonaxisymmetric mode prevails for finite Biot number and axisymmetric mode becomes the only mode for infinite Biot number.

(2) The number of mode change with respect to aspect ratio decreases when Biot number raises.

(3) For fixed aspect ratio, the critical Rayleigh number increases as Biot number enhances due to the allowance of heat leaving across the sidewall.

\section{Acknowledgment}

This work is funded by Ministry of Science and Technology R.O.C. under grant No. MOST 103-2221-E-260 $-028-\mathrm{MY} 2$

\section{References}

[1] C. W. Horton and F. T. Rogers, "Convection currents in a porous medium," J. Appl. Phys., Vol. 16, pp. 367-370, 1945.

[2] E. R. Lapwood, "Convection of a fluid in a porous medium," Math. Proc. Camb. Philos. Soc., Vol. 44, pp. 508-521, 1948.

[3] J. L. Beck, "Convection in a box of porous material saturated with fluid," Phys. Fluids, Vol. 15, pp. 1377-1383, 1972.

[4] A. Zebib, "Onset of natural convection in a cylinder of water saturated porous medium," Phys. Fluids, Vol. 21, pp. 699-670, 1978.

[5] C. Y. Wang, "Onset of natural convection in a fluid-saturated porous medium inside a cylindrical enclosure bottom heated by a constant flux,” Int. Comm. Heat Mass Transfer, Vol. 25, pp. 593-598, 1998.

[6] H. H. Bau and K. E. Torrance, "Low Rayleigh number thermal convection in a vertical cylinder filled with porous materials and hated from below," J. Heat Transfer, Vol. 104, 166-172, 1982.

[7] C. Y. Wang, "Thermo-convective stability of a fluid- saturated porous medium inside a cylindrical enclosure - Permeable top constant flux heating," Mech. Res. Comm., Vol. 26, pp. 603-608, 1999.

[8] J. P. Kubitschek and P. D. Weidman, "Stability of a fluid-saturated porous medium contained in a vertical cylinder heated from below by force convection," Heat Mass Transfer, Vol. 42, pp. 789-794, 2006.

[9] K. B. Haugen and P. A. Tyvand, "Onset of thermal convection in a vertical porous cylinder with conducting wall,” Phys. Fluids, Vol. 15, pp. 2661-2667, 2003.

[10] T. Nilsen and L. Storesletten, "An analytical study on natural convection in isotropic and anisotropic porous channels," Trans. ASME J. Heat Transfer, Vol. 112, pp. 396-401, 1990.

[11] D. A. S. Rees and J. L. Lage, "The effect of thermal stratification on natural convection in a vertical porous insulation layer," Int. J. Heat Mass Transfer, Vol. 40, pp. 111-121, 1997

About Author (s):

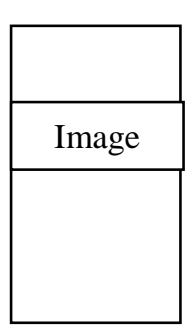

Prof. I-Chung Liu got his Bachelor degree at Department of Naval Architecture, National Taiwan University, Taiwan, R.O.C. on 1988, Master degree on 1990, and $\mathrm{Ph} . \mathrm{D}$. on 1994. His present research of interest fall in the category of Fluid

Dynamic, Heat Transfer, Hydraulics, and Disaster Mitigation.
Table 1 Mode change sequence $(m, n)$ and the corresponding $\left(R a_{\mathrm{c}}, a\right)$ from slender cylinder $a \approx 0$ to wide cylinder $a \approx 3$.

\begin{tabular}{|c|c|c|c|c|c|c|c|c|c|}
\hline$B i=10^{-4}$. & $(1,1)$ & $\begin{array}{l}\rightarrow \\
\rightarrow \\
\rightarrow\end{array}$ & $\begin{array}{l}(2,1) \\
(1,2) \\
(6,1) \\
(4,2) .\end{array}$ & $\begin{array}{l}\rightarrow \\
\rightarrow \\
\rightarrow\end{array}$ & $\begin{array}{l}(0,1) \\
(5,1) \\
(3,2)\end{array}$ & $\begin{array}{l}\rightarrow \\
\rightarrow \\
\rightarrow\end{array}$ & $\begin{array}{l}(3,1) \\
(2,2) \\
(1,3)\end{array}$ & $\begin{array}{l}\rightarrow \\
\rightarrow \\
\rightarrow\end{array}$ & $\begin{array}{l}(4,1) \cdot \\
(0,2) . \\
(7,1) .\end{array}$ \\
\hline$\left(R a_{s}, a\right)$ & \multicolumn{3}{|c|}{$\begin{array}{l}(0.751 .41 .9303) \\
(1.693 .39 .4789) \\
(2.310 .39 .5222) \\
(2.841 .39 .5394)\end{array}$} & $\begin{array}{l}.089,39 \\
862,39 . \\
.469 .39\end{array}$ & $\begin{array}{l}9798) \\
8166) \\
5211)\end{array}$ & \multicolumn{2}{|c|}{$\begin{array}{l}(1.278,39.5603) \\
(2.088,39.4979) \\
(2.633 .39 .5177)\end{array}$} & \multicolumn{2}{|c|}{$\begin{array}{l}(1.505,40.0265) \\
(2.184,39.4891) \\
(2.726 .39 .4790)\end{array}$} \\
\hline$B i=0.1$. & $(1,1)$ & $\begin{array}{l}\rightarrow \\
\rightarrow \\
\rightarrow\end{array}$ & $\begin{array}{l}(2,1) \\
(5,1) \\
(1,3) \\
\end{array}$ & $\begin{array}{l}\rightarrow \\
\rightarrow \\
\rightarrow\end{array}$ & $\begin{array}{l}(0,1) \\
(2,2) \\
(4,1) .\end{array}$ & $\begin{array}{l}\rightarrow \\
\rightarrow\end{array}$ & $\begin{array}{l}(3,1) \\
(0,2)\end{array}$ & $\begin{array}{l}\rightarrow \\
\rightarrow\end{array}$ & $\begin{array}{l}(1,2) .1 \\
(3,2)\end{array}$ \\
\hline$\left(R a_{s}, a\right)$ & $\begin{array}{l}(0.7 \\
(1.89 \\
(2.6) \\
\end{array}$ & $\begin{array}{l}51.42 .9 \\
95.40 .1 \\
17.39 .6 \\
\end{array}$ & $\begin{array}{ll}9042) & (1.0 \\
1872) & (1.9 \\
6712) & (2.8\end{array}$ & $\begin{array}{l}.066,40.5 \\
921,40.1 \\
841,39.6\end{array}$ & $\begin{array}{l}5177) \\
1120) \\
6907)\end{array}$ & $\begin{array}{l}(1.328,40 . \\
(2.169 .39 .6\end{array}$ & 0774) & $\begin{array}{l}(1.479 .40 \\
(2388.39\end{array}$ & $\begin{array}{l}\text { 4635).. } \\
\text { 8198).. }\end{array}$ \\
\hline$B i=1.1$ & $(1,1)$ & $\begin{array}{l}\rightarrow \\
\rightarrow \\
\rightarrow\end{array}$ & $\begin{array}{l}(2,1) \\
(0,2) \\
(2,3) .\end{array}$ & $\begin{array}{l}\rightarrow \\
\rightarrow\end{array}$ & $\begin{array}{l}(0,1) \\
(3,1)\end{array}$ & $\begin{array}{l}\rightarrow \\
\rightarrow\end{array}$ & $\begin{array}{l}(1,2) \\
(1,3)\end{array}$ & $\begin{array}{l}\rightarrow \\
\rightarrow\end{array}$ & $\begin{array}{l}(2,2) . \\
(4,1) .\end{array}$ \\
\hline$\left(R a_{s}, a\right)$ & $\begin{array}{l}(0.72 \\
(2.08 \\
(2.9)\end{array}$ & $\begin{array}{l}29.49 .5 \\
82.40 .6 \\
50.40 .2\end{array}$ & $\begin{array}{ll}5336) & (0.8 \\
6762) & (2.4 \\
2757) & \end{array}$ & $\begin{array}{l}893,459 \\
402,40.5\end{array}$ & $\begin{array}{l}9494) \\
5775)\end{array}$ & $\begin{array}{l}(1.413 .42 \\
(2.528,40\end{array}$ & $\begin{array}{l}3741) \\
\text { 4291) }\end{array}$ & $\begin{array}{l}(1.884,41 \\
(2.895,40\end{array}$ & $\begin{array}{l}2628) . .1 \\
2990) . .\end{array}$ \\
\hline$B i=10 .$. & $(1,1)$ & $\begin{array}{l}\rightarrow \\
\rightarrow\end{array}$ & $\begin{array}{l}(0,1) \\
(0,3) \cdot .\end{array}$ & $\rightarrow$ & $(1,2)$ & $\rightarrow$ & $(0,2)$ & $\rightarrow$ & $(1,3)$ \\
\hline$\left(R a_{s}, a\right)$ & & $\begin{array}{l}83.73 .0 \\
13.40 .6 \\
\end{array}$ & $\begin{array}{l}761) \\
6159) \\
61.4\end{array}$ & $422,44.1$ & $1294)($ & $(1.868 .422$ & 2415) & $(2.455,41.0$ & $0642) \cdot$ \\
\hline$B i=10^{s}$. & $\begin{array}{l}\text { All mod } \\
1.637< \\
\end{array}$ & $\begin{array}{l}\text { des are } \\
a<1.6\end{array}$ & $\begin{array}{l}\text { axisymm } \\
63 \text { and } 2.6\end{array}$ & $\begin{array}{l}\text { metric es } \\
.673<a\end{array}$ & $\begin{array}{l}\text { scept fo } \\
-2.703 .\end{array}$ & for azimu & & es $m=1$ & aregions \\
\hline $\mathrm{Bi} \rightarrow$ & Allm & & isy? & & & & & & \\
\hline
\end{tabular}

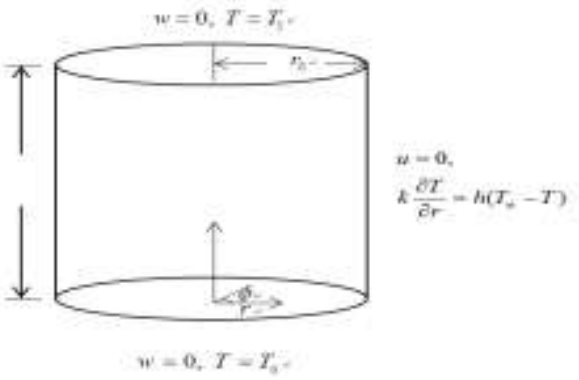

Figure 1. Physical configuration and coordinates system.

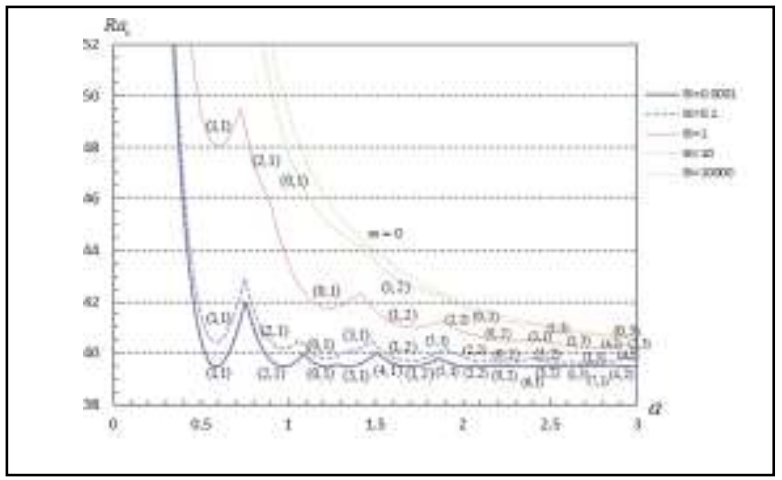

Figure 2 Stability curves versus $a$ for various $B i$.

About Author (s)

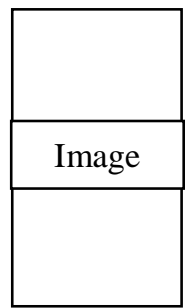

Master Yen-Tsung Chang presently serves as a Ph.D. candidate and works as a senior Technician in Center of Environmental Protection, Safety and Health, National Chi Nan University. 